\title{
OPEN Investigation of skin microbiota reveals Mycobacterium ulcerans-Aspergillus sp. trans-kingdom communication
}

\author{
N. Hammoudi ${ }^{1,2}$, C. Cassagne ${ }^{3}$, M. Million ${ }^{2}$, S. Ranque $\mathbb{1}^{3}$, O. Kabore ${ }^{2}$, M. Drancourt ${ }^{2}$, \\ D. Zingue ${ }^{2,4}$ \& A. Bouam ${ }^{2 \bowtie}$
}

Mycobacterium ulcerans secrete a series of non-ribosomal-encoded toxins known as mycolactones that are responsible for causing a disabling ulceration of the skin and subcutaneous tissues named Buruli ulcer. The disease is the sole non-contagion among the three most common mycobacterial diseases in humans. Direct contact with contaminated wetlands is a risk factor for Buruli ulcer, responsible for $M$. ulcerans skin carriage before transcutaneous inoculation with this opportunistic pathogen. In this study, we analysed the bacterial and fungal skin microbiota in individuals exposed to M. ulcerans in Burkina Faso. We showed that $M$. ulcerans-specific DNA sequences were detected on the unbreached skin of $6 / 52(11.5 \%)$ asymptomatic farmers living in Sindou versus $0 / 52(0 \%)$ of those living in the non-endemic region of Tenkodogo. Then, we cultured the skin microbiota of asymptomatic $M$. ulcerans carriers and negative control individuals, all living in the region of Sindou. A total of 84 different bacterial and fungal species were isolated, 21 from $M$. ulcerans-negative skin samples, 31 from $M$. ulcerans-positive samples and 32 from both. More specifically, Actinobacteria, Aspergillus niger and Aspergillus flavus were significantly associated with $M$. ulcerans skin carriage. We further observed that in vitro, mycolactones induced spore germination of $A$. flavus, attracting the fungal network. These unprecedented observations suggest that interactions with fungi may modulate the outcome of $M$. ulcerans skin carriage, opening new venues to the understanding of Buruli ulcer pathology, prophylaxis and treatment of this still neglected tropical infection.

Buruli ulcer is a disabling chronic disease of the skin and the subcutaneous tissues ${ }^{1}$.

The disease has been reported since 1948 in several tropical regions ${ }^{2}$, with the highest incidence being observed in West African countries, including Côte d'Ivoire, Ghana, and Benin ${ }^{3}$. The causative agent Mycobacterium ulcerans is a non-tuberculous mycobacterium harbouring a $174-\mathrm{kb}$ circular virulence plasmid $\mathrm{pMUM}^{4,5}$ encoding three genes, $m l s \mathrm{~A} 1, m l s \mathrm{~A} 2$ and $m l s \mathrm{~B}$, responsible for the non-ribosomal synthesis of mycolactone toxins, the main virulence factors of the pathogen ${ }^{6}$. This macrolide exotoxin is secreted by a group of closely related non-tuberculous mycobacteria named Mycolactone Producing Mycobacteria (MPM) ${ }^{7,8}$. Comparative analysis of whole genome sequences showed that MPM form a single clonal group that evolved from a Mycobacterium marinum parent ${ }^{9}$. This group is divided into three lineages, including frog and fish pathogens in one lineage ${ }^{10,11}$, the Japanese strain $M$. ulcerans subsp. shinshuense in a second lineage, while the third lineage includes a highly clonal group responsible for Buruli ulcer in Africa and Australia ${ }^{9}$. Every strain synthesizes at least one of the eight congeners of mycolactone A/B named mycolactone C, D, E, F, dia-F, S1 and S2 ${ }^{12}$. Each type of mycolactone exhibits a variable degree of cytotoxicity, with mycolactone A/B being the most biologically active ${ }^{13}$. Indeed, this macrolide toxin is responsible for cytotoxic effects, namely, apoptosis and necrosis, in addition to immunosuppressive and analgesic effects ${ }^{12}$ after M. ulcerans has penetrated breached skin to initiate discrete oedematous lesions that can evolve into typical Buruli ulcer lesions ${ }^{14}$. Currently, the mode of transmission of M. ulcerans is debated. While it is clear that M. ulcerans must be inoculated through the skin to elicit Buruli ulcer, the specific role of aquatic bugs and mosquitoes as potential vectors is debated along with the possibility of passive entry by any skin breach regardless of its cause. It is not known whether M. ulcerans can colonize skin in asymptomatic

${ }^{1}$ IHU Méditerranée Infection, Marseille, France. ${ }^{2}$ IRD, MEPHI, IHU Méditerranée Infection, Aix Marseille Univ., 19-21 Bd Jean Moulin, 13385 Marseillle Cedex 05, France. ${ }^{3}$ IRD, AP-HM, SSA, VITROME, IHU Méditerranée Infection, Aix Marseille Univ., Marseille, France. ${ }^{4}$ Centre Muraz, Bobo-Dioulasso, Burkina Faso. ${ }^{\circledR}$ email: amarbouam@yahoo.fr 
populations exposed to environments contaminated with $M$. ulcerans. Mycobacteria of the M. ulcerans group are known as environmental organisms residing in poorly defined aquatic ecological niches where they could be part of an alimentary chain ${ }^{15}$. We previously reported that $M$. ulcerans could thrive in environmental niches containing bacteria, fungi, algae and mollusks with which $M$. ulcerans may exchange nutrients ${ }^{16}$. One may hypothesize that the cutaneous microbiota, which is part of the individual, partially controls the expression of the M. ulcerans infection ${ }^{17}$. Indeed, bacteria and fungi present on skin contaminated with M. ulcerans could interact with the pathogen, as previously shown with the antagonism between Staphylococcus lugdunensis and Staphylococcus aureus in the nasal mucosa ${ }^{18,19}$.

To contribute to this medical debate, we have undertaken the first study to evaluate the possibility of asymptomatic carriage of $M$. ulcerans on healthy skin, to characterize the live cutaneous bacterial and fungal microbiota associated with the asymptomatic carriage of M. ulcerans, using culturomics.

\section{Methods \\ Sample collection. Sampling was performed in two regions of Burkina Faso. Sindou is located in the rural district of Niofila, Douna Department, Province of Léraba in the Cascades region in the southwest of Burkina Faso near the borders with Côte d'Ivoire and Mali. People sampled in this region are farmers in frequent contact with stagnant water because of their daily activities in rice, banana and vegetable cultivation areas that are irri- gated from a nearby dam. Cases of Buruli ulcer have already been reported in this region ${ }^{20}$. Tenkodogo, a town located in the province of Boulgou and the Central-Eastern region of Burkina Faso, was used as a negative con- trol region free of Buruli ulcer. Additionally, in this region, farmers working near two dams in rice and vegetable cultures were swabbed (the lower legs of the farmers were cleaned with water and then swabbed with sterile swabs (Deltalab, Barcelona, Spain) containing $1 \mathrm{~mL}$ of sterile $0.9 \%$ sodium chloride solution, the swabbed parts did not contain any visible lesions.). A total of 104 farmers were sampled, 52 farmers in each region. In Sindou region, the average age was 37 years, 30 females and 22 males. In Tenkodogo region the male/female ratio was $27 / 25$ respectively with an average age of 20 years. One sample was taken per individual. Samples were stored at $4{ }^{\circ} \mathrm{C}$ after collection in Burkina Faso, transported to IHU Méditerannée Infection laboratory, Marseille, France at ambient temperature and stored at $4{ }^{\circ} \mathrm{C}$ before performing analysis.}

Detection of M. ulcerans DNA. Real-time PCR amplifications. Total DNA from the samples and from a six-week-old culture of $M$. ulcerans CU001 (positive control) was extracted using a QIAamp Tissue Kit by QUIAGEN-BioRobot EZ1, according to the manufacturer's instructions (Qiagen, Hilden, Germany). To assess PCR inhibition, $10 \mu \mathrm{L}$ of an external control was added to $190 \mu \mathrm{L}$ of sample volume as previously described ${ }^{21}$. Extracted DNA was used in real-time PCR (RT-PCR) to amplify the insertion sequences (IS2404 and IS2606) and the ketoreductase-B domain of the mycolactone polyketide synthase genes (KR-B), using RT-PCR reagents from Roche PCR Kit (Roche Diagnostics, Meylan, France) and primers and probes as previously described ${ }^{22}$ in a CFX $96^{\text {Tix }}$ real-time PCR thermocycler and detection system (BIO-Rad, Marnes-la-Coquette, France). To estimate M. ulcerans inoculum in skin swabs, we performed three calibration curves for the IS2404, IS2606, and KR-B genes. The total DNA of the M. ulcerans CU001 strain calibrated at $1 \mathrm{McFarland}=10^{6} \mathrm{CFU}$ was extracted with EZ (Qiagen, Hilden, Germany). Then, tenfold serial dilutions of up to $10^{-8}$ were made to generate a calibration curve for each system. Two reactional mixes were incorporated into each PCR run as negative controls. Samples were considered positive when the KR-B gene was detected with $\mathrm{Ct}<40$ cycles, and at least one of the two PCRs of the insertion sequence gave $\mathrm{Ct}<40$ cycles and KR-B was detected with $\mathrm{Ct}<40$ cycles.

Skin microbiota repertoire. Bacterial culture. All RT-PCR-positive samples collected in Sindou were selected for culture. Six negative samples collected in the same region were used as negative controls. For each sample, a cascade dilution (up to $10^{-10}$ ) was performed in sterile PBS (Thermo Fisher Diagnostics, Dardilly, France), and $100 \mu \mathrm{L}$ of each dilution was inoculated in duplicate on blood agar plates (bioMérieux, Marcy l'Etoile, France). One blood agar plate was incubated aerobically, and the second was anaerobically incubated at $37^{\circ} \mathrm{C}$ (an incubation temperature chosen to mimic environmental temperature in the Burkinabe area where sampling was done) for $72 \mathrm{~h}$. After incubation, each colony presenting a unique morphology was sub-cultured onto a blood agar plate and incubated at $37^{\circ} \mathrm{C}$ for $48 \mathrm{~h}$ under the appropriate atmosphere to isolate each colony type.

Fungal culture. M. ulcerans PCR-positive samples were cultured in three different culture media, namely, homemade RMI medium, Sabouraud agar (Oxoid, Dardilly, France) and Chromagar (Becton Dickinson, Le Pont de Claix, France) incubated at $30^{\circ} \mathrm{C}$ for seven days. Each colony was sub-cultured and incubated under the same conditions. The six PCR-negative samples (negative controls) were treated using the same protocol.

Matrix assisted laser desorption ionization time-of-flight identification of colonies. The identification of the bacterial and fungal colonies was carried out using matrix-assisted laser desorption ionization time-of-flight mass spectrometry MALDI-TOF-MS as previously described ${ }^{23,24}$. For bacteria, each colony was deposited in duplicate onto a MALDI-TOF MSP 96 target plate (Bruker Daltonics, Leipzig, Germany), and $2 \mu \mathrm{L}$ of matrix solution (saturated solution of alpha-cyano-4- hydroxycinnamic acid in $50 \%$ acetonitrile and $2.5 \%$ trifluoroacetic acid) was added to each spot and allowed to dry for $5 \mathrm{~min}$ and then analysed by Microflex spectrometer (Bruker Daltonics) using the software MALDI BioTyper 3.0 (Bruker Daltonics). For the identification of fungi, each colony was incubated in $1 \mathrm{~mL}$ of $70 \%$ ethanol for $10 \mathrm{~min}$ and then centrifuged at $1300 \mathrm{~g}$ for $5 \mathrm{~min}$. The pellet was treated with $20 \mu \mathrm{L}$ of acetonitrile and formic acid (v.v) at $100 \%$ and $70 \%$, respectively. This mixture was then centrifuged at $1300 \mathrm{~g}$ for $5 \mathrm{~min}$, and $1.5 \mu \mathrm{L}$ of the supernatant was deposited on a MALDI-TOF-MS target and allowed to dry 
before $1.5 \mu \mathrm{L}$ of matrix was deposited on each spot, allowed to dry for $5 \mathrm{~min}$ and then analysed by par Microflex spectrometer (Bruker Daltonics) using the software MALDI BioTyper 3.0 (Bruker Daltonics).

Molecular identification and sequencing. All colonies that remained unidentified by MALDI-TOF-MS were subjected to molecular identification by sequencing the bacterial $16 \mathrm{~S}$ rRNA $^{25}$ and the fungal ITS1, ITS2, $\beta$ tubulin and TEF regions. The primers used in this study are reported in Table S1. DNA extraction was performed using BioRobot EZ1 (Qiagen, Les Ullis, France) using the commercial EZ1 DNA Tissue Kit according to the manufacturer's instructions (Qiagen). PCR was performed using Hotstar Taq polymerase according to the manufacturer's instructions (Qiagen) using a thermocycler (Applied Biosystem, Paris, France). PCR products were separated by electrophoresis on a $1.5 \%$ agarose gel and stained with SYBR ${ }^{\star}$ safe (Thermo Fisher Scientific) before being visualized under an ultraviolet transilluminator. PCR products were then purified using a Millipore NucleoFast 96 PCR kit following the manufacturer's recommendations (Macherey-Nagel, Düren, Germany) and sequenced using the BigDye Terminator Cycle Sequencing Kit (Applied Biosystems) with an automatic sequencer ABI (Applied Biosystems). Sequences were assembled using the software ChromasPro 1.7 (Technelysium Pty Ltd., Tewantin, Australia) and blasted in the NCBI databank using default settings to identify bacterial species $^{26}$ and against the Mycobank database http://www.mycobank.org/ to identify fungal species.

Fungi-M. ulcerans biological interactions. The three fungal species found to significantly correlate with the presence/absence of $M$. ulcerans skin carriage (A. flavus, A. niger and P. rubens) were cultured in the presence/ absence of mycolactones $\mathrm{AB} / \mathrm{C}$ extracted from a culture of $M$. ulcerans CU001 to observe the effect of mycolactones on spore germination and the fungal network. Briefly, agar plates were cut into T-shape, the fungal spores were placed on the middle strand, and two virgin absorbent paper discs were placed on each side of the plates. $20 \mu \mathrm{L}$ of PBS were put on the first disc (negative control) and $20 \mu \mathrm{L}$ of mycolactones $\mathrm{AB} / \mathrm{C}$ on the second disc. The chemoattractant effect of mycolactone $\mathrm{AB} / \mathrm{C}$ was measured as previously described ${ }^{27}$. On the other hand, fungal spores were put in Sabouraud liquid medium containing $20 \mathrm{uL}$ of mycolactones $\mathrm{AB} / \mathrm{C}$ or $20 \mu \mathrm{L}$ of $\mathrm{PBS}$ as a negative control. The effect of Mycolactone $\mathrm{AB} / \mathrm{C}$ on spore germination was then monitored as previously described $^{27}$.

Statistical analyses. We first compared the proportion of positive cutaneous samples for M. ulcerans in Buruli ulcer-endemic region and Buruli ulcer-free region by a two-sided exact Fisher's test. We then explored the association between $M$. ulcerans and cutaneous bacterial and fungal species by comparing the microbiota of 6 positive samples and 6 random negative samples from the same region. To do so, we primarily performed an unsupervised analysis of the cutaneous bacterial and fungal microbiota using a main component analysis by integrating the presence or absence of each bacterium or fungus detected in at least one individual and adding the variable "detection of M. ulcerans by PCR". We complemented this analysis with a supervised comparison between individuals positive and negative for M. ulcerans by PCR. To do this, we compared the detection frequency in the two groups. Statistical significance was calculated by the two-sided exact Fisher's test. Owing to the possible risk of missing important findings, adjustments for multiple comparisons were not performed, as suggested for exploratory work ${ }^{28}$. A double-clustering heatmap was used to visualize the potential clustering of cultured fungal and bacterial repertoires with M. ulcerans-positive or negative skin samples. Finally, we carried out a discriminant factor analysis to test whether the fungal skin microbiota discriminated against individuals who were positive or negative for M. ulcerans skin carriage. The principal component and discriminant factor analyses were performed using XLSTAT v2019.1 statistical and data analysis solution (Long Island, NY, USA (https://www.xlstat.com).

Multivariate analysis was carried out on the set of microbes cultured from M. ulcerans positive and negative samples to reveal possible associations between some microbes and the positivity of $M$. ulcerans skin carriage. Multivariate analysis was performed via the Genmod procedure with the SAS 9.4 statistical software using a negative binomial distribution, adjusted for the effect of time (quantitative variable) and by using generalized estimating equations to account for the non-independence of repeated measures to analyze the results of germination assays. The Wilcoxon signed rank test and Chi-squared test were used to compare the attraction effect of fungi by mycolactones and to compare M. ulcerans prevalence in the two geographical regions of Sindou and Tenkodogo, respectively. Statistical tests were performed on the website biostaTGV, https://biostatgv.sentiweb.fr.

Ethics approval. This study was approved by the Centre MURAZ Ethics Committee, Burkina Faso, and reference 2018-11/MS/SG/CM/CEI. All research was performed in accordance with relevant guidelines and regulations.

Consent to participate. All adult subjects provided informed oral consent, and a parent or guardian of any child participant provided informed oral consent on the child's behalf after explaining the merits of the study. Written consent could not be obtained because the study was conducted among an illiterate population. The research presents no more than minimal risk of harm to subjects and involves no procedures for which written consent is normally required outside of the research context (Sec. 56.109 IRB review of research).

\section{Results}

M. ulcerans DNA detection. Negative controls introduced in every batch of real-time PCR remained negative, and positive controls were positive for all the targeted sequences IS2404, IS2606 and KR-B, allowing the establishment of calibration curves. Of the 52 swabs collected in the region of Sindou, eight were positive 


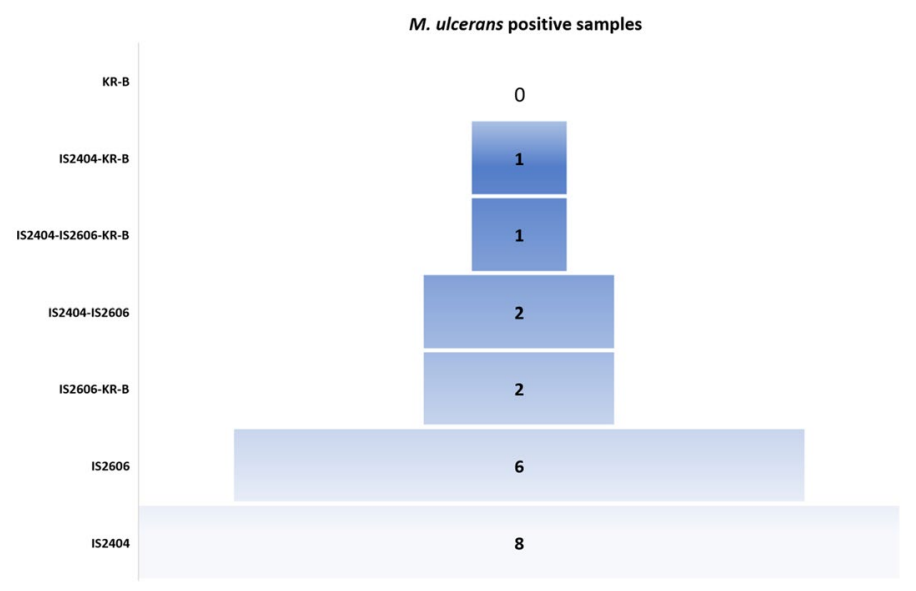

Figure 1. Real-time PCR detection of M. ulcerans from 52 swabs collected in the Sindou region, IS2404 and IS2606 are specific insertion sequences for M. ulcerans KR-B ketoreductase-B gene.

for IS2404, six were positive for IS2606, two were positive for both IS2404 and IS2606, one was positive for both IS2404 and KR-B, two were positive for both IS2606 and KR-B, one was positive for all three targeted sequences and 32 samples were negative (Fig. 1). Therefore, 6/52 (11.5\%) swabs were positive for at least two M. ulcerans DNA sequences. As expected, only three samples were positive for IS2404 only in Tenkodogo, so none of the 52 skin samples collected in this negative control region were positive for $M$. ulcerans, confirming the probable absence of $M$. ulcerans in this region. The difference in the prevalence of RT-PCR-based detection of $M$. ulcerans between the two regions was significant ( $p=0.04, \mathrm{~N}-1$ Chi-squared test). Based on the calibration curves established during this study, the Ct values observed here were extrapolated to 9-50 colony forming units (CFU) of M. ulcerans.

Skin microbiota identification. A total of 84 different species of microorganisms (62 bacterial and 22 fungal species) belonging to 45 different genera were recovered from the twelve samples. Bacteria included 20 (32\%) Gram-negative species and 42 (68\%) Gram-positive species. Thirty-one different species of microorganisms (19 bacterial and 12 fungal species) grew exclusively in M. ulcerans-PCR positive samples. Thirty-two different species (22 bacterial and 10 fungal species) grew only from $M$. ulcerans-PCR negative samples. Twentyone bacterial species grew on both $M$. ulcerans-PCR positive samples and $M$. ulcerans-PCR negative samples. No fungal species were found in both types of samples simultaneously (Fig. 2). Globally, the microbiota of $M$. ulcerans-PCR positive samples was statistically enriched for Actinobacteria (Fisher's test $p$ value $=0.03$ ). The genus Aspergillus and the species Aspergillus flavus were significantly associated with $M$. ulcerans-PCR positive samples (two-sided Fisher's exact test $p$ value $=0.0021$ and 0.015 , respectively), and the genus Penicillium was significantly associated with $M$. ulcerans-PCR negative samples ( $p$ value $=0.0021$, Fisher's exact test). All Zygomycetes, Acidovorax, Brevundimonas, Cutibacterium, and Homoserinibacter species were recovered from PCR M. ulcerans-positive samples, whereas all Penicillium, Cellulosimicrobium, Franconibacter, Ochrobactrum, Porphyromonas, Roseomonas, Achromobacter and Lelliottia species were recovered from M. ulcerans-PCR-negative samples, but these associations were not statistically significant (Fig. 2). The dendrogram obtained by the agglomerative hierarchical classification of the species recovered from samples harboured two distinct clusters perfectly separating the M. ulcerans-PCR positive samples from the M. ulcerans-PCR negative samples (Fig. 3). These results highly suggest that the skin microbiota was significantly correlated with $M$. ulcerans carriage.

Principal component analysis. After submission of the results of fungal and bacterial culturomics and PCR M. ulcerans to PCA analysis, two varifactors (F1 and F7; representing both a total of $28.3 \%$ of the variance of the data) were selected because these two varifactors represented the highest variance of the variable "PCR M. ulcerans". F1 and F7 contributed $20.4 \%$ and $7.9 \%$ of the overall variability, respectively. Aspergillus flavus, Propionimicrobium lymphophilum and Propionimicrobium freudenreichii were strongly associated with a positive PCR for M. ulcerans, whereas Penicillium rubens, Penicillium chrysogenum and Lelliota nimipressuralis were associated with a negative PCR. Surprisingly, detection of Aspergillus fungi positively correlated with PCR detection of $M$. ulcerans. Indeed, A. flavus was the fungus exhibiting the strongest positive association with $M$. ulcerans. On the other hand, and unexpectedly, we observed that the detection of Penicillium fungi was anticorrelated with the detection of M. ulcerans. No Penicillium detection positively correlated with M. ulcerans detection, while P. rubens and P. chrysogenum were the only fungi to anti-correlate with M. ulcerans (Fig. 4).

Factor discriminant analysis. Interestingly, factor discriminant analysis identified five fungal and bacterial species that significantly discriminated $M$. ulcerans-PCR positive and negative samples, including A. flavus, Brevundimonas diminuta, Propionimicrobium lymphophilum, Pantoea dispersa and P. rubens ( $p$ values: 0.001, $0.01,0,049,0.049,0.049$, respectively-Table S2). The discriminant analysis showed that the cutaneous fungal 


\begin{tabular}{|c|c|c|c|}
\hline Species & Relative frequency difference & $P$ value & Species \\
\hline Aspergillus flavus & & $*$ & Klebsiella variicola \\
\hline Aspergillus niger & & & Enterococcus malodoratus \\
\hline Acidovorax temperans & & & Staphylococcus epidermidis \\
\hline Brevundimonas diminuta & & & Bacillus cereus \\
\hline Cellulomonas massiliensis & & & Lactobacillus plantarum \\
\hline Propionimicrobium lymphophilum & & & Bacillus dielmoensis \\
\hline Microbacterium paraoxydans & & & Bacillus megaterium \\
\hline Microbacterium oleivorans & & & Clostridium subterminale \\
\hline Propionibacterium freudenreichii & & & Stenotrophomonas maltophild \\
\hline Micrococcus luteus & & & Propionibacterium acnes \\
\hline Pseudomonas aeruginosa & & & Cryptococcus laurentii \\
\hline Pantoea dispersa & & & Gymnascella aurantiaca \\
\hline Bacillus marasmi & & & Pochonia chlamydosporia \\
\hline Paenibacillus alvei & & & Exophiala xenobiotica \\
\hline Staphylococcus capitis & & & Sordariomycete sp \\
\hline Lactobacillus reuteri & & & Penicillium commune \\
\hline Bacillus pumilus & & & Dothideomycete \\
\hline Pichia carribica & & & Microbacterium paludicola \\
\hline Cutibacterium acnes & & & Cellulomonas iranensis \\
\hline Microbacterium hydrocarbonoxydans & & & Cellulosimicrobium cellulans \\
\hline Microbacterium testaceum & & & Franconibacter pulveris \\
\hline Homoserinibacter gongjuensis & & & Pantoea terrea \\
\hline Purpureocillium lilacinum & & & Pantoea anthophila \\
\hline Aspergillus nidulans & & & Ochrobactrum haemophilum \\
\hline Aspergillus tubingensis & & & Roseomonas gilardii \\
\hline Curtobacterium citreum & & & Pseudomonas stutzeri \\
\hline Microbacterium arborescens & & & Pantoea calida \\
\hline Ruminococcus merdae & & & Bacillus benzoevorans \\
\hline Lodderomyces elongisporus & & & Bacillus niacini \\
\hline Arxula adeninivorans & & & Bacillus subtilis \\
\hline Ogataea thermomethanolica & & & Bacillus drentensis \\
\hline Exophiala spinifera & & & Bacillus paralicheniformis \\
\hline Syncephalastrum racemosum & & & Porphyromonas uenonis \\
\hline Rhizopus oryzae & & & Penicillium crustosum \\
\hline Acinetobacter Iwoffii & & & Micrococcus yunnanensis \\
\hline Microbacterium lacticum & & & Achromobacter spanius \\
\hline Cellulomonas parahominis & & & Paenibacillus yonginensis \\
\hline Microbacterium neinengenese & & & Bacillus marisflavi \\
\hline Collinsella massiliensis & & & Staphylococcus hominis \\
\hline Klebsiella pneumoniae & & & Penicillium rubens \\
\hline Enterobacter cloacae & & & Penicillium chrysogenum \\
\hline Pantoea stewartii & & & Lelliottia nimipressuralis \\
\hline
\end{tabular}

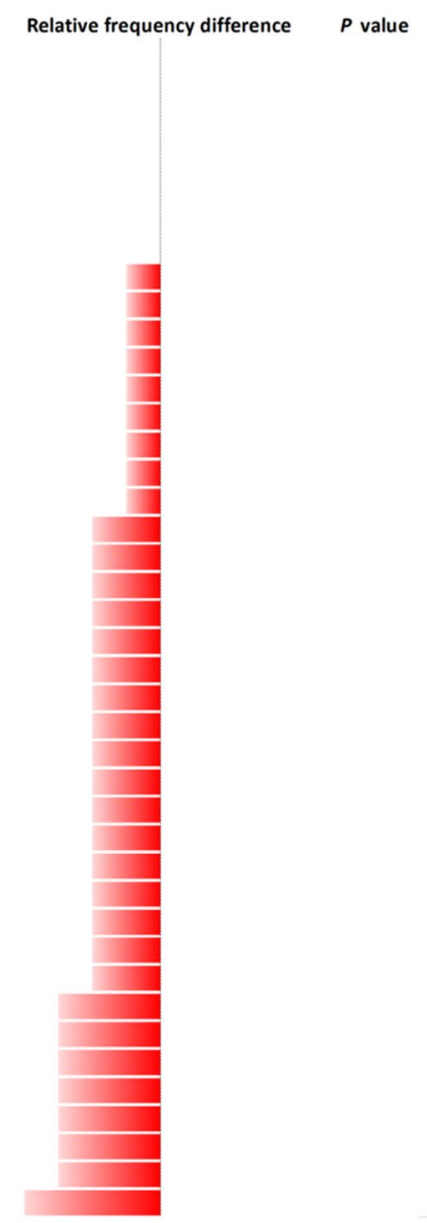

Figure 2. The different microorganisms grown from 12 skin samples comprising 6 M. ulcerans positive samples and 6 negative samples are presented with their relative frequency differences and associated $p$ values. A total of 84 species distributed among 62 bacteria and 22 fungi were cultured.

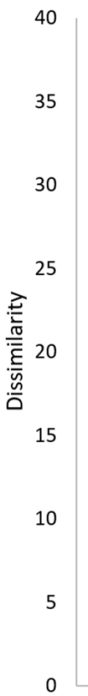

\section{Dendrogram}

a

Positive samples
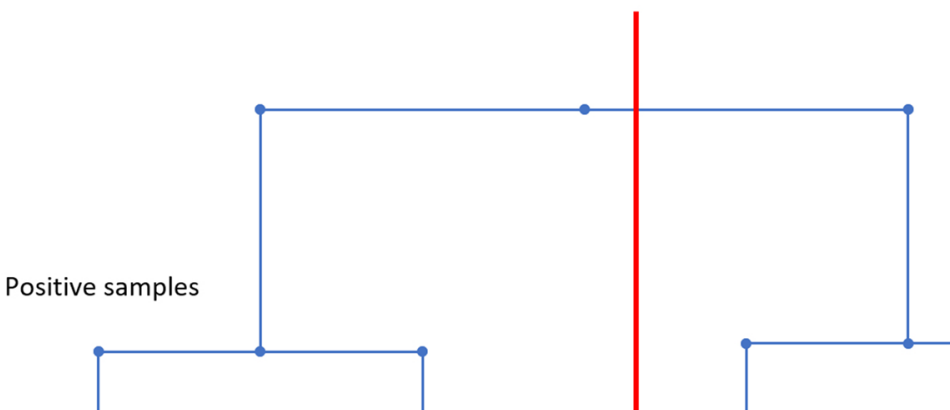

Figure 3. The agglomerative hierarchical classification dendrogram of individuals according to their cutaneous microbiota associated with the detection (P1-P6) or absence (N1-N6) of M. ulcerans. M. ulcerans PCR-negative samples and $M$. ulcerans PCR-positive samples were clearly separated into two distinct clusters according to the composition of the skin microbiota. 


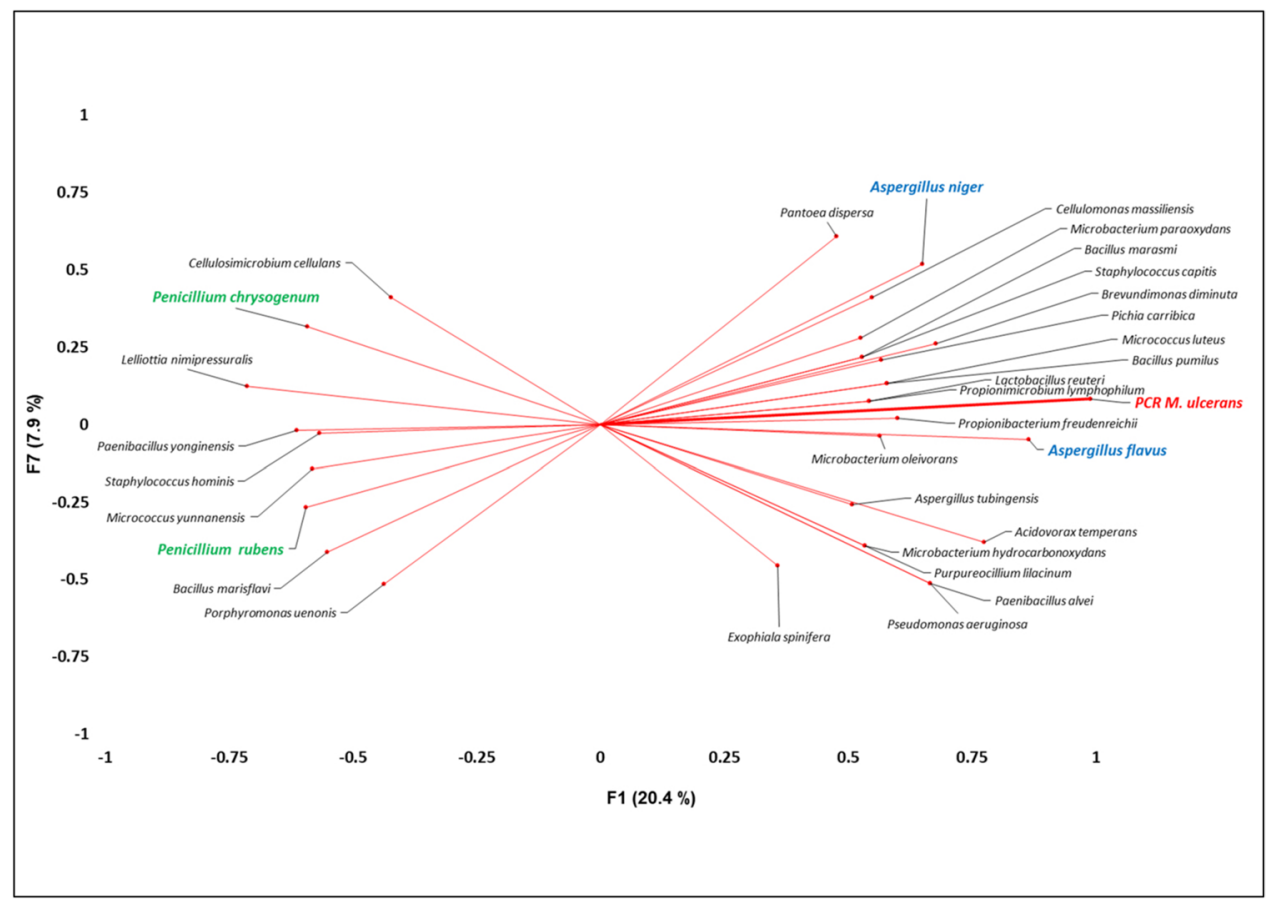

Figure 4. PCA of 84 bacterial and fungal species isolated from individuals in Burkina Faso revealed a strong association of A. flavus, P. lymphophilum and P. freudenreichii with M. ulcerans skin carriage.

and bacterial microbiota discriminated with $100 \%$ accuracy between $M$. ulcerans positive and negative groups (receiver operator curve analysis: area under curve $=1-$ no error in the confusion matrix - perfect clustering).

Fungi-M. ulcerans biological interactions. Mycolactones significantly increased spore germination of A. flavus after a 10 -h incubation (stimulated $91.2 \%$ vs. control $28.75 \% ; p<0.0001$ ), significantly increased spore germination of $A$. niger (stimulated $75.42 \%$ vs. control $69.17 \% ; p<0.0001$ ), and significantly decreased spore germination of $P$. rubens (stimulated $45.2 \%$ vs. control 50.7\%; $p<0.0001$ ) (Fig. 5). Moreover, we observed that mycolactones significantly attracted A. flavus and A. niger during the T-test assay ( $p$ value: 0.013 and 0.003 , respectively) (Fig. 6).

\section{Discussion}

Using a standard RT-PCR assay to detect the asymptomatic skin carriage of $M$. ulcerans in skin samples collected from individuals living in Burkina Faso, we detected a prevalence of $11.5 \%$ of $M$. ulcerans carriage in individuals residing in the Sindou region, Burkina Faso, where Buruli ulcer cases have been previously reported ${ }^{20}$. Our data agree with a previous report of cutaneous carriage of $M$. ulcerans in Ghana among people practicing agriculture without any protective clothing and in infants crawling on the soil ${ }^{29}$. Thus, the data reported here confirm that in Buruli ulcer-endemic areas, some people are asymptomatic skin carriers of M. ulcerans. The M. ulcerans inoculum we detected on the skin has been reported to be sufficient to initiate Buruli ulcer lesions in a murine model $^{30}$. These observations suggest that asymptomatic skin carriage could be a previously undescribed condition in the natural history of Buruli ulcer.

Exploration of the skin microbiota at the interface of healthy and diseased skin is in its infancy, especially regarding Buruli ulcer ${ }^{31}$. Here, we used culture to address this question because we were interested in representing the live microbiota to provide the bacterial and fungal strains to assess their biological interactions with $M$. ulcer$a n s^{27}$. Indeed, it has been showed than metagenomics has less discriminant power and is unable to differentiate live or dead microbes ${ }^{32-34}$ and we felt that some of the metagenomics limits (under-representation of minority populations partially due to bias in DNA extraction, limits in accurate species identification) could be partially overcome by using culturomics, an experimental approach which contributed to increase the number of species isolated from humans at $66.2 \%{ }^{35}$. Exploring the exhaustivity of the bacterial and fungal skin repertoire in studied population was not an aim of this study and accordingly, the particular culture conditions we used may have limited growth of some microbes. Nevertheless, the comparison of skin microbiota between M. ulcerans-PCR positive and negative samples revealed a specific cutaneous microbiota associated with asymptomatic M. ulcerans skin carriage, even predicting $M$. ulcerans skin carriage. Other skin pathogens and diseases (leprosy and psoriasis) have been previously related to a specific skin microbiota ${ }^{35,36}$. The novelty in our study was to find significant, antiparallel associations between $M$. ulcerans and fungi: $M$. ulcerans was detected on the skin along with Aspergillus, in which spore germination and the fungal network were stimulated by mycolactones, whereas the detection of M. ulcerans has never been associated with the presence of Penicillium species in the skin, Accordingly, spore germination of $P$. rubens was inhibited by mycolactones. Therefore, our clinical observations were not fortuitous 

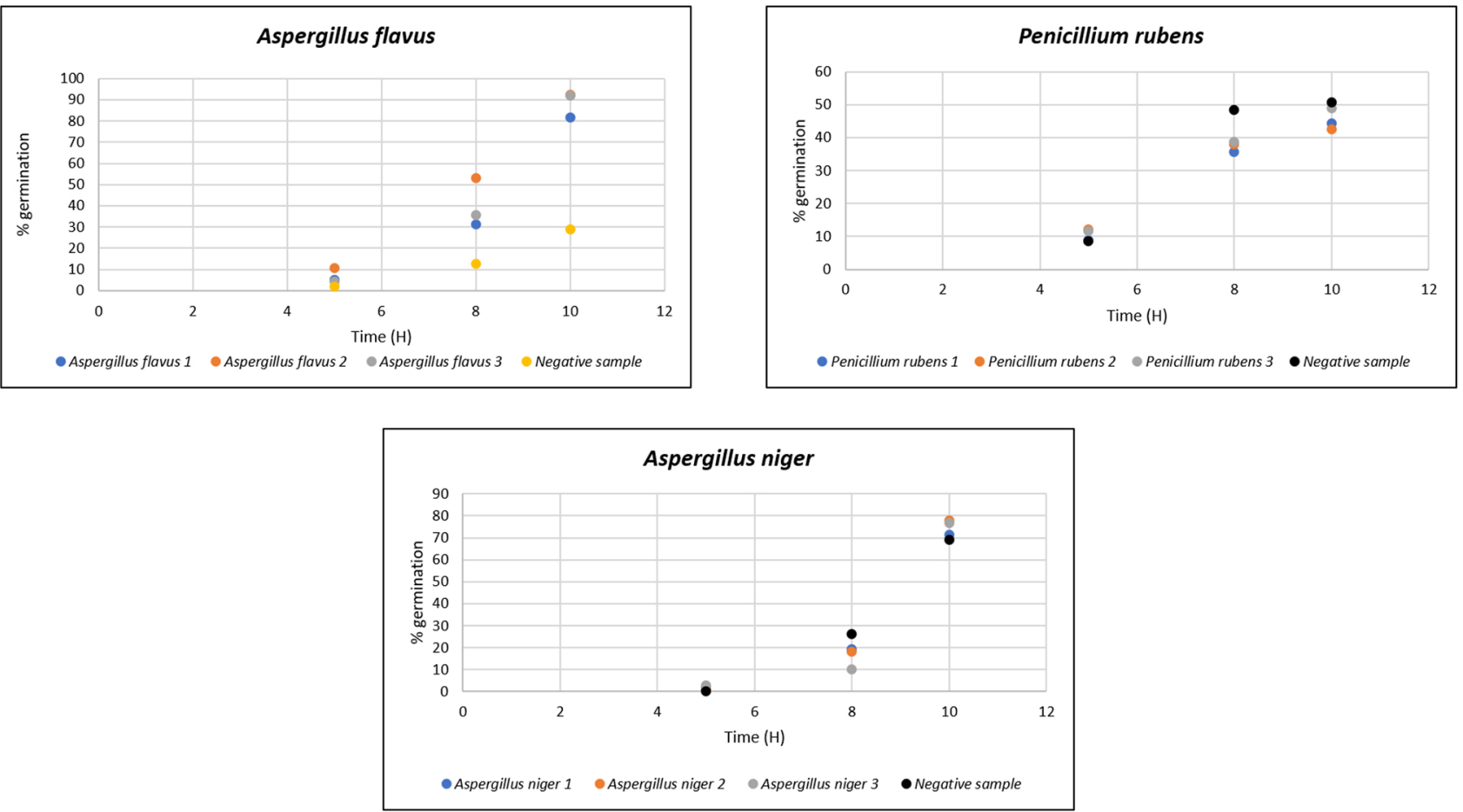

Figure 5. Mycolactone-induced spore germination of A. flavus, A niger and P. rubens in the presence of PBS as a negative control.
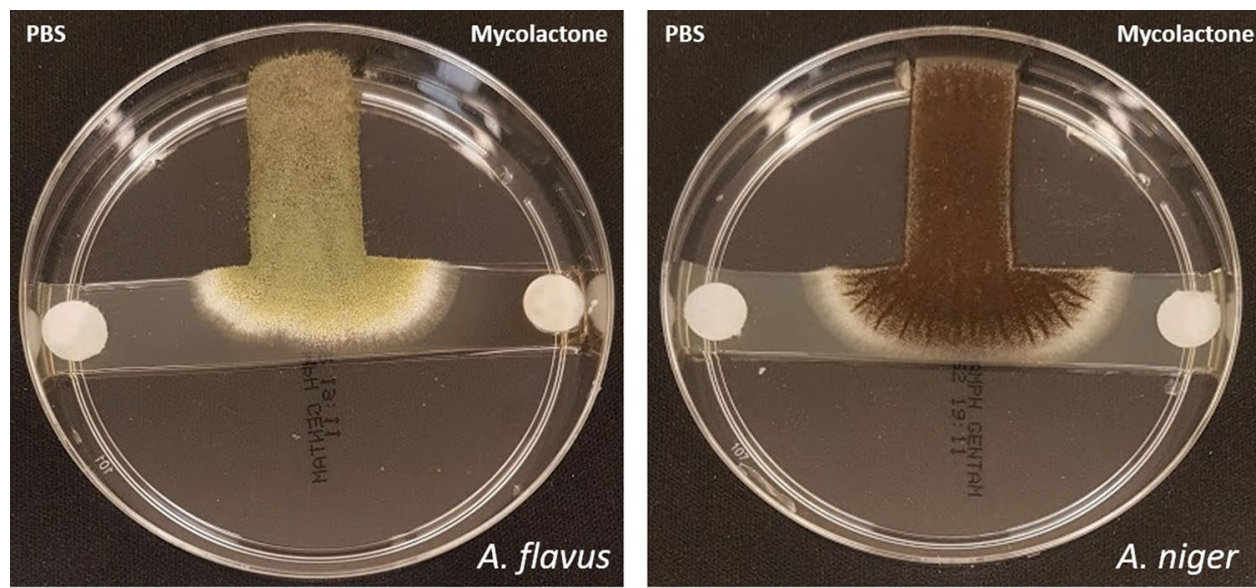

Figure 6. Fungi attraction test: mycolactones (right side disk) attract A. flavus and A. niger in the presence of PBS as a negative control (left side disk).

but revealed transkingdom mycobacteria-fungi interactions, supporting preliminary observations made with the Zygomycete $M$ ucor circinelloides ${ }^{27}$. Of note, the transkingdom communication concerned $P$. rubens, a penicillinproducing strain, alias P. chrysogenum, made famous by Sir Alexander Fleming ${ }^{37}$.

All these observations suggest for the first time that transkingdom communications between fungi and mycobacteria are of medical interest, partially driving the natural history of Buruli ulcer. These observations will stimulate additional studies to disclose whether this holds true for other mycobacterial infections of medical interest.

\section{Data availability}

All sequences of bacteria and fungi identified in this study were deposited in NCBI, accession numbers are available from Table S3. MALDI-TOF spectra are also available from https://doi.org/10.6084/m9.figshare.13483290.v1.

Received: 3 January 2020; Accepted: 7 January 2021

Published online: 12 February 2021 


\section{References}

1. MacCallum, P., Tolhurst, J. C., Buckle, G. \& Sissons, H. A. A new mycobac- terial infection in man. J. Pathol. Bacteriol. 60, 93-102. https://doi.org/10.1002/path.1700600111 (1948).

2. Johnson, P. D. et al. Buruli ulcer (M. ulcerans infection): new insights, new hope for disease control. PLoS Med. 2, e108 (2005).

3. Brou, T., Broutin, H., Elguero, E., Asse, H. \& Guegan, J. F. Landscape diversity related to Buruli ulcer disease in Cote d'Ivoire. PLoS Negl. Trop. Dis. 2, e271 (2008).

4. George, K. M. et al. Mycolactone: a polyketide toxin from Mycobacterium ulcerans required for virulence. Science 283, 854-857 (1999).

5. Stinear, T. P. et al. Giant plasmid-encoded polyketide synthases produce the macrolide toxin of Mycobacterium ulcerans. Proc. Natl. Acad. Sci. USA 101, 1345-1349 (2004).

6. Stinear, T. P. et al. Reductive evolution and niche adaptation inferred from the genome of Mycobacterium ulcerans, the causative agent of Buruli ulcer. Genome Res. 17, 192-200 (2007).

7. Pidot, S. J., Asiedu, K., Käser, M., Fyfe, J. A. \& Stinear, T. P. Mycobacterium ulcerans and other mycolactone-producing mycobacteria should be considered a single species. PLoS Negl. Trop. Dis. 7, e663 (2010).

8. Yoshida, M. et al. Complete genome sequence of Mycobacterium ulcerans subsp. shinshuense. Genome Announc. 4, 1050-1116 (2016).

9. Doig, K. D. et al. On the origin of Mycobacterium ulcerans, the causative agent of Buruli ulcer. BMC Genomics 13, 258 (2012).

10. Ucko, M. et al. Strain variation in Mycobacterium marinum fish isolates. Appl. Environ. Microbiol. 68, 5281-5287 (2002).

11. Stragier, P., Hermans, K., Stinear, T. \& Portaels, F. First report of a mycolactone-producing Mycobacterium infection in fish agriculture in Belgium. FEMS Microbiol. Lett. 286, 93-95 (2008).

12. Gehringer, M. \& Altmann, K. H. The chemistry and biology of mycolactones. Beilstein J. Org. Chem. 3, 1596-1660 (2017).

13. Mve-Obiang, A., Lee, R. E., Portaels, F. \& Small, P. L. Heterogeneity of mycolactones produced by clinical isolates of Mycobacterium ulcerans: implications for virulence. Immun. Infect. 71, 774-783 (2003).

14. George, K. M., Pascopella, L., Welty, D. M. \& Small, P. L. A Mycobacterium ulcerans toxin, mycolactone, causes apoptosis in guinea pig ulcers and tissue culture cells. Infect. Immun. 68, 877-883 (2000).

15. Morris, A. L. et al. Deforestation-driven food-web collapse linked to emerging tropical infectious disease, Mycobacterium ulcerans. Sci. Adv. 2, 1600387 (2016).

16. Zingue, D., Bouam, A., Tian, R. B. D. \& Drancourt, M. Buruli ulcer, a prototype for ecosystem-related infection, caused by $M y c o-$ bacterium ulcerans. Clin. Microbiol. Rev. 31, 45-17 (2018).

17. Van Leuvenhaege, C. et al. Bacterial diversity in Buruli ulcer skin lesions: challenges in the clinical microbiome analysis of a skin disease. PLoS ONE 12, 0181994 (2017).

18. Lina, G. et al. Bacterial competition for human nasal cavity colonization: role of staphylococcal agr alleles. Appl. Environ. Microbiol. 69, 18-23 (2003)

19. Amissah, N. A. et al. Genetic diversity of Staphylococcus aureus in Buruli ulcer. PLoS. Negl. Trop. Dis. 9, 3421 (2015).

20. Ouoba, K. et al. Buruli ulcers in Burkina Faso: apropos of 6 cases. Tunis Med. 76, 46-50 (1998).

21. Ninove, L. et al. RNA and DNA bacteriophages as molecular diagnosis controls in clinical virology: a comprehensive study of more than 45,000 routine PCR tests. PLoS ONE 6, 16142 (2011).

22. Fyfe, J. A. et al. Development and application of two multiplex real-time PCR assays for the detection of Mycobacterium ulcerans in clinical and environmental samples. Appl. Environ. Microbiol. 73, 4733-4740 (2007).

23. Seng, P. et al. MALDI-TOF-mass spectrometry applications in clinical microbiology. Future Microbiol. 5, 1733-1754 (2010).

24. Cassagne, C., Normand, A. C., Lollivier, C., Ranque, S. \& Piarroux, R. Performance of MALDI-TOF MS platforms for fungal identification. Mycoses 59, 678-90 (2016)

25. Drancourt, M. Outils moléculaires d'identification en bactériologie. Med. Mal Infect. 28, 380-382 (1998).

26. Altschul, S. F., Gish, W., Miller, W., Myers, E. W. \& Lipman, D. J. Basic local alignment search tool. J. Mol. Biol. 215, 403-410 (1990).

27. Hammoudi, N. et al. Mycobacterium ulcerans mycolactones-fungi crosstalking. Sci Rep. 9, 3028 (2019).

28. Rothman, K. J. No adjustments are needed for multiple comparisons. Epidemiology 1, 43-46 (1990).

29. Prah, I., Examination of human skin surfaces for the detection of Mycobacterium ulcerans. M.Sc. Thesis. University of Ghana.. Available from: http://ugspace.ug.edu.gh/handle/123456789/8369.

30. Wallace, J. R. et al. Mycobacterium ulcerans low infectious dose and mechanical transmission support insect bites and puncturing injuries in the spread of Buruli ulcer. PLoS Negl. Trop. Dis. 11, 5553 (2017).

31. Cho, I. \& Blaser, M. J. The human microbiome at the interface of health and disease. Nat. Rev. Genet. 13, 260-270 (2012).

32. Lagier, J. C. et al. Culturing the human microbiota and culturomics. Nat. Rev. Microbiol. 1, 540-550 (2018).

33. Pham, T. P. et al. Gut microbiota alteration is characterized by a proteobacteria and fusobacteria bloom in kwashiorkor and a bacteroidetes paucity in marasmus. Sci. Rep. 9, 9084 (2019).

34. Seck, E. H. et al. Salt in stools is associated with obesity, gut halophilic microbiota and Akkermansia muciniphila depletion in humans. Int. J. Obes. (Lond). 43, 862-871 (2019).

35. Silva, P. E. et al. Insights into the skin microbiome dynamics of leprosy patients during multi-drug therapy and in healthy individuals from Brazil. Sci. Rep. 8, 8783 (2018).

36. Fahlén, A., Engstrand, L., Baker, B. S., Powles, A. \& Fry, L. Comparison of bacterial microbiota in skin biopsies from normal and psoriatic skin. Arch. Dermatol. Res. 304, 15-22 (2012).

37. Houbraken, J., Frisvad, J. C. \& Samson, R. A. Fleming's penicillin producing strain is not Penicillium chrysogenum but P. rubens. IMA Fungus 2, 87-95 (2011)

\section{Acknowledgements}

The authors acknowledge the technical help of Nicholas Armstrong. This study was supported by IHU Méditerranée Infection, Marseille, France and by the French Government under the «Investissements d'avenir» (Investments for the Future) program managed by the Agence Nationale de la Recherche (ANR, fr: National Agency for Research), (reference: Méditerranée Infection 10-IAHU- 03). This work was supported by Région Provence Alpes Côte d'Azur and European funding FEDER PRIMI.

\section{Author contributions}

N.H.: performed the experiments, interpreted the data and drafted the manuscript. C.C.: conceived and performed the experiments, interpreted the data and drafted the manuscript. M.M.: performed statistical analyses, interpreted the data and drafted the manuscript. S.R.: conceived the experiments, interpreted the data and drafted the manuscript. K.O.: performed sampling, collected epidemiological data and drafted the manuscript. Z.D.: performed some experiments, interpreted the data and drafted the manuscript. M.D.: conceived the experiments, interpreted the data and drafted the manuscript. A.B.: performed some experiments, conceived the experiments, interpreted the data and drafted the manuscript. 


\section{Competing interests}

The authors declare no competing interests.

\section{Additional information}

Supplementary Information The online version contains supplementary material available at https://doi. org/10.1038/s41598-021-83236-7.

Correspondence and requests for materials should be addressed to A.B.

Reprints and permissions information is available at www.nature.com/reprints.

Publisher's note Springer Nature remains neutral with regard to jurisdictional claims in published maps and institutional affiliations.

(c) (1) Open Access This article is licensed under a Creative Commons Attribution 4.0 International License, which permits use, sharing, adaptation, distribution and reproduction in any medium or format, as long as you give appropriate credit to the original author(s) and the source, provide a link to the Creative Commons licence, and indicate if changes were made. The images or other third party material in this article are included in the article's Creative Commons licence, unless indicated otherwise in a credit line to the material. If material is not included in the article's Creative Commons licence and your intended use is not permitted by statutory regulation or exceeds the permitted use, you will need to obtain permission directly from the copyright holder. To view a copy of this licence, visit http://creativecommons.org/licenses/by/4.0/.

(c) The Author(s) 2021 